\title{
INFRARED LUMINOUS LYMAN BREAK GALAXIES: A POPULATION THAT BRIDGES LBGs AND SCUBA GALAXIES
}

\author{
J.-S. Huang, ${ }^{1}$ D. Rigopoulou, ${ }^{2}$ S. P. Willner,${ }^{1}$ C. Papovich, ${ }^{3}$ C. Shu, ${ }^{4,5}$ M. L. N. Ashby, ${ }^{1}$ P. Barmby, ${ }^{1}$ \\ K. Bundy, ${ }^{6}$ C. Conselice, ${ }^{6}$ E. Egami, ${ }^{3}$ P. G. Pérez-González, ${ }^{3}$ J. L. Rosenberg, ${ }^{1}$ \\ H. A. Smith, ${ }^{1}$ G. Wilson, ${ }^{7}$ and G. G. Fazio ${ }^{1}$ \\ Received 2005 May 27; accepted 2005 July 28
}

\begin{abstract}
A deep mid- and far-infrared survey in the extended Groth strip (EGS) area gives 3.6 to $8 \mu \mathrm{m}$ flux densities or upper limits for 253 Lyman break galaxies (LBGs). The LBGs are a diverse population but with properties correlated with luminosity. The LBGs show a factor of 30 range in indicated stellar mass and a factor of 10 range in apparent dust content relative to stellar mass. About 5\% of LBGs are luminous at all wavelengths, with powerful emission at rest $6 \mu \mathrm{m}$. In the rest 0.9 to $2 \mu \mathrm{m}$ spectral range these galaxies have stellar spectral slopes with no sign of an AGN power-law component, suggesting that their emission is mainly powered by intensive star formation. Galaxies in this luminous population share the infrared properties of cold Submillimeter Common-User Bolometric Array (SCUBA) sources: both are massive and dusty starburst galaxies at $2<z<3$; their stellar mass is larger than $10^{11} M_{\odot}$. We suggest that these galaxies are the progenitors of present-day giant elliptical galaxies, with a substantial fraction of their stars already formed at $z \approx 3$.
\end{abstract}

Subject headings: cosmology: observations — galaxies: high-redshift — infrared: galaxies — surveys

\section{INTRODUCTION}

The two most efficient ways of identifying galaxies at high redshifts are (1), the Lyman break broadband dropout technique, and (2), observations at submillimeter wavelengths. For example, surveys with SCUBA on the James Clerk Maxwell Telescope reveal high-redshift objects via emission from the cold dust they contain (Ivison et al. 2002; Smail et al. 2002; Chapman et al. 2003). Conventional wisdom is that the submillimeter observations preferentially select starburst galaxies at $z>2$ because the far-infrared luminosity peak shifts into the submillimeter band (Ivison et al. 2002; Smail et al. 2002; Chapman et al. 2003). Most SCUBA sources are too faint in the optical and near-infrared bands for spectroscopic identification, but Chapman et al. (2003) and Simpson et al. (2004) have confirmed that most SCUBA sources in their sample are at $z>2$. Despite all the effort that has gone into SCUBA searches, Lyman break galaxies (LBGs) still constitute by far the largest well-studied rest-frame UV-selected galaxy samples at high redshift (Steidel et al. 2003).

The relation between LBGs and SCUBA galaxies remains unclear, but recently Chapman et al. (2005) have confirmed that some SCUBA galaxies have rest-frame UV colors typical of LBGs at $z \sim 2$ (Steidel et al. 2004). Understanding both populations requires a direct comparison of properties, such as stellar mass and dust emission. The four bands viewed by the Infrared Array

\footnotetext{
${ }^{1}$ Harvard-Smithsonian Center for Astrophysics, 60 Garden Street, Cambridge, MA 02138.

2 Department of Astrophysics, Oxford University, Keble Road, Oxford, OX1 3RH, UK

${ }^{3}$ Steward Observatory, University of Arizona, Tucson, AZ 85721.

4 Joint Center for Astrophysics, Shanghai Normal University, Shanghai 200234, China.

5 Shanghai Astronomical Observatory, Chinese Academy of Sciences, Shanghai 200030, China.

6 California Institute of Technology, MS 105-24, 1201 East California, Pasadena, CA 91125.

7 Spitzer Science Center, California Institute of Technology, 1200 East California, Pasadena, CA 91125.
}

Camera (IRAC) aboard Spitzer, from 3.6 to $8.0 \mu \mathrm{m}$, cover spectral energy distributions (SEDs) for galaxies at $2<z<3$ in the rest-frame near-infrared, where the luminosities are good indicators of stellar mass (Cole et al. 2001; Drory et al. 2004; Glazebrook et al. 2004; Bundy et al. 2005). The Multiband Imaging Photometer for Spitzer (MIPS) $24 \mu \mathrm{m}$ band is an ideal probe of the polycyclic aromatic hydrocarbon (PAH) and hot dust emission from LBGs and SCUBA galaxies. Preliminary studies show that Spitzer can easily detect both LBGs and SCUBA galaxies (Barmby et al. 2004; Egami et al. 2004; Huang et al. 2004).

This paper presents the first study of a large LBG sample in the extended Groth strip (EGS) region based on Spitzer observations carried out with deep IRAC and MIPS photometry. We report the discovery of a new type of LBG, the infrared-luminous LBG (ILLBG) with strong infrared emission at rest frame $6 \mu \mathrm{m}$, whose properties are very similar to those of SCUBA galaxies. ILLBGs are massive, dusty, and powered mainly by extremely rapid star formation.

\section{SPITZER OBSERVATIONS}

IRAC (Fazio et al. 2004a, 2004b) observations of the EGS were carried out in two epochs, 2004 January and June, covering a $2^{\circ} \times 10$ strip. The MIPS observations were done in 2004 June in scanning mode. Limiting flux densities $(5 \sigma)$ are roughly $0.5 \mu \mathrm{Jy}$ at 3.6 and $4.5 \mu \mathrm{m}, 2.7 \mu \mathrm{Jy}$ at 5.8 and $8.0 \mu \mathrm{m}$, and $60 \mu \mathrm{Jy}$ at $24 \mu \mathrm{m}$. We use the same data-reduction procedure for both IRAC and MIPS data as Huang et al. (2004) and Egami et al. (2004).

The LBG sample in the EGS area was taken from Steidel et al. (2003). Among a total of 334 objects in the Steidel et al. (2003) catalog, 188 are spectroscopically identified as galaxies at $z \sim 3$, 3 are QSOs, 3 are AGNs, and 7 are stars. The remaining $133 \mathrm{ob}-$ jects are not identified. In the photometric catalog, 253 objects are in the area covered by both IRAC and MIPS imaging. Of these, 211 are detected at $3.6 \mu \mathrm{m}, 199$ at $4.5 \mu \mathrm{m}, 53$ at $5.8 \mu \mathrm{m}$, and 44 at $8.0 \mu \mathrm{m}$. We detect 11 LBG counterparts in the MIPS 


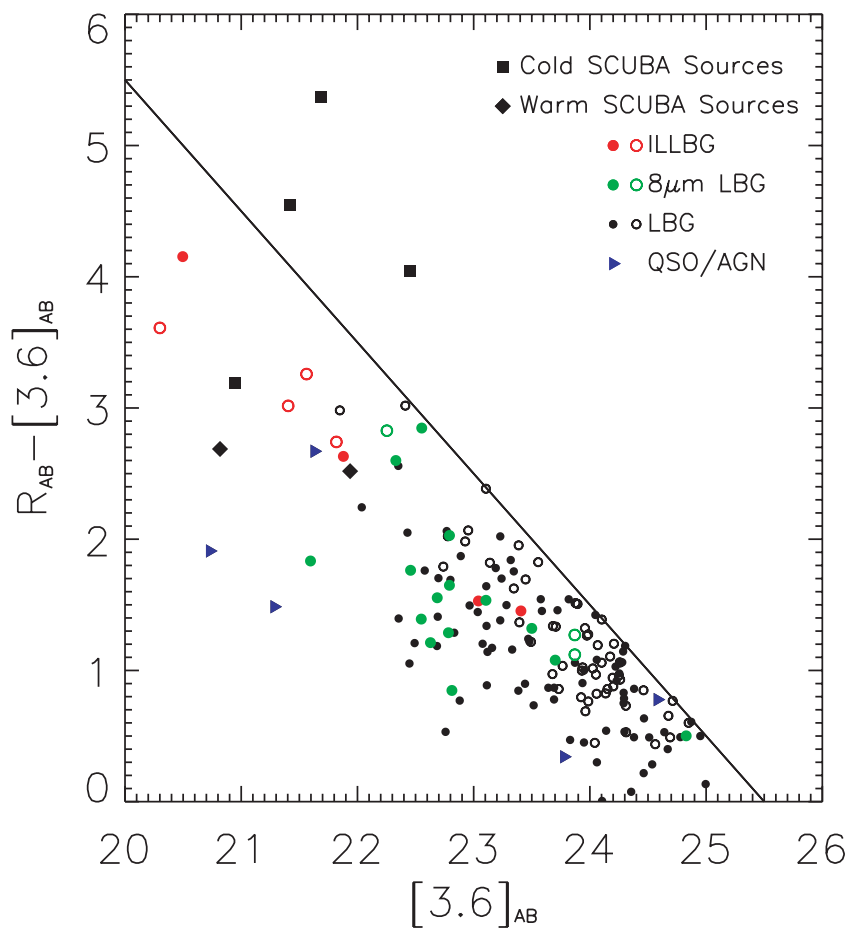

FIG. 1.-Observed-frame $R$-[3.6] vs. [3.6] color-magnitude diagram for all LBGs in our EGS sample. Black circles indicate LBGs detected only at 3.6 and $4.5 \mu \mathrm{m}$. The green circles are LBGs detected at $8 \mu \mathrm{m}$ but not $24 \mu \mathrm{m}$, and red circles are LBGs detected in both 8 and $24 \mu \mathrm{m}$ bands. For all these objects, filled symbols designate sources having spectroscopic redshifts, while open symbols are for objects with photometric redshifts only. Filled squares and diamonds represent the cold and warm SCUBA sources in the Lockman Hole (Egami et al. 2004). The straight line is the $R$-band limiting magnitudes of $R=25.5$ (Steidel et al. 2003).

$24 \mu \mathrm{m}$ image, including all 3 QSOs and 1 AGN identified by Steidel et al. (2003). ${ }^{8}$ The $24 \mu$ m-detected counterparts are also detected at all shorter wavelengths and are among the brightest sources in the sample. We define the LBGs with $24 \mu \mathrm{m}$ detections $\left(f_{24 \mu \mathrm{m}}>60 \mu \mathrm{Jy}\right)$ as ILLBGs. The $24 \mu \mathrm{m}$ limiting flux is equivalent to $L_{6.2 \mu \mathrm{m}}=6 \times 10^{8} L_{\odot} \mu \mathrm{m}^{-1}$ for galaxies at $z=3$, or $L_{\mathrm{IR}}=3 \times 10^{10} L_{\odot}$ if we use the M82 SED to convert the luminosity at $6.2 \mu \mathrm{m}$ to the total infrared luminosity.

We compare the LBG sample with a set of Lockman Hole area SCUBA sources having secure identifications (Huang et al. 2004; Egami et al. 2004). ${ }^{9}$ Egami et al. (2004) classified these SCUBA sources into two types (cold and warm) based on the dust temperature inferred from their SEDs. The cold sources exhibit the $1.6 \mu \mathrm{m} \mathrm{H}^{-}$opacity minimum bump in the IRAC bands, a stellar feature typically seen in galaxy SEDs. The cold SCUBA sources are well approximated by the SED of Arp 220, a cold dusty starburst. The warm sources are AGN dominated, with a power-law continuum SED, similar to that of Mrk 231. There

\footnotetext{
${ }^{8}$ Two additional LBGs may be detected at $24 \mu \mathrm{m}$, but there are non-LBG galaxies within $3^{\prime \prime}$ of them on the sky. Given the angular resolution of MIPS, we cannot be sure which objects are responsible for the $24 \mu \mathrm{m}$ flux, and to be conservative we do not count these two as $24 \mu \mathrm{m}$ LBG detections.

${ }^{9}$ Finding counterparts to SCUBA sources is difficult because there are many faint optical and infrared sources within the SCUBA error circle. For reliable identifications, radio observations are needed to give accurate positions. Four SCUBA sources in the EGS IRAC+MIPS area have good radio observations (Webb et al. 2003), but none of the counterparts thereby identified has a known redshift. In fact, SEDs suggest that at least two of the four are at $z<2$. Thus the EGS SCUBA sources do not provide a useful comparison sample, and we compare the EGS LBGs with the Lockman Hole SCUBA sources instead.
}

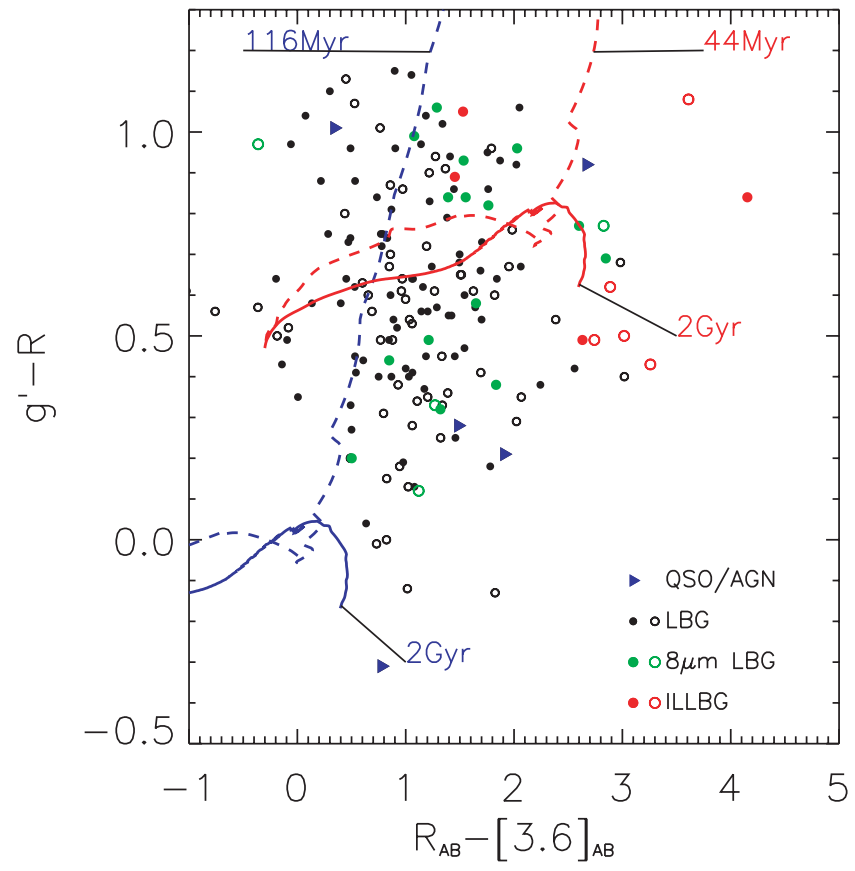

FIG. 2.-Observed frame $g-R$ vs. $R-[3.6]$ color-color diagram for all LBGs detected with IRAC. Symbols are as in Fig. 1. The lines show colors from stellar synthesis models: dashed lines are the single-burst models, and solid lines are the constant star formation models. The blue lines are dust-free models, and the red lines are models with $E(B-V)=0.3$ and a Calzetti et al. (2000) reddening law. The models run from $0.1 \mathrm{Myr}$ at the blue end of the lines for the reddened models, off the plot for the dust-free models to $2 \mathrm{Gyr}$ as indicated for the constant star formation models, above the top of the plot range for the single-burst models. Maximum ages for the single-burst models are indicated near the top of the figure. Overall the set of models spans nearly the entire color range of the data.

are four cold and two warm sources in the Lockman Hole SCUBA sample (Egami et al. 2004; Huang et al. 2004).

\section{INFRARED COLORS OF LBGs}

The LBGs exhibit a much wider range of flux densities in the IRAC bands than in the $K$ band. Figure 1 shows that the range of $3.6 \mu \mathrm{m}$ flux densities for the LBGs spans $4 \mathrm{mag}$, compared to only $1.5 \mathrm{mag}$ for the range of $K$-band flux density seen by Shapley et al. (2001). Figure 1 also shows a correlation between [3.6] and $R-$ [3.6] for LBGs. This relation is partially due to a selection effect (all LBGs are brighter than $R=25.5$; Steidel et al. 2003), but there is an obvious absence of LBGs with blue colors at higher $3.6 \mu \mathrm{m}$ flux densities. All ILLBGs (as defined above) are redder than $R-[3.6]=1.5$, and most have $R-[3.6]>2.4$. Three ILLBGs are so red that they are close to qualifying as extremely red objects (EROs) $(R-[3.6]>4$; Wilson et al. 2004). LBGs detected at $8 \mu \mathrm{m}$ are redder than other galaxies $(\langle R-[3.6]\rangle=1.95$ vs. 0.94 for LBGs detected at $3.6 \mu \mathrm{m}$ but not $8 \mu \mathrm{m}$ ), but not so red as the ILLBGs. All six Lockman Hole SCUBA sources have red $R-[3.6]$ colors (Wilson et al. 2004), comparable to those of the ILLBGs.

Figure 2 compares the observed LBG colors with those predicted by two simple stellar population synthesis models (single burst and constant star formation). These two models correspond to two extreme cases of a model having an exponentially decaying star formation rate, $\psi(t)=e^{-t / \tau}$, where $\tau=0$ is the single-burst model and $\tau=\infty$ is the constant star formation model. For any given $E(B-V)$, the constant star formation model predicts the reddest $R-[3.6]$ color for galaxies at $z \sim 3$. A combination of the two models with varying amounts of dust 


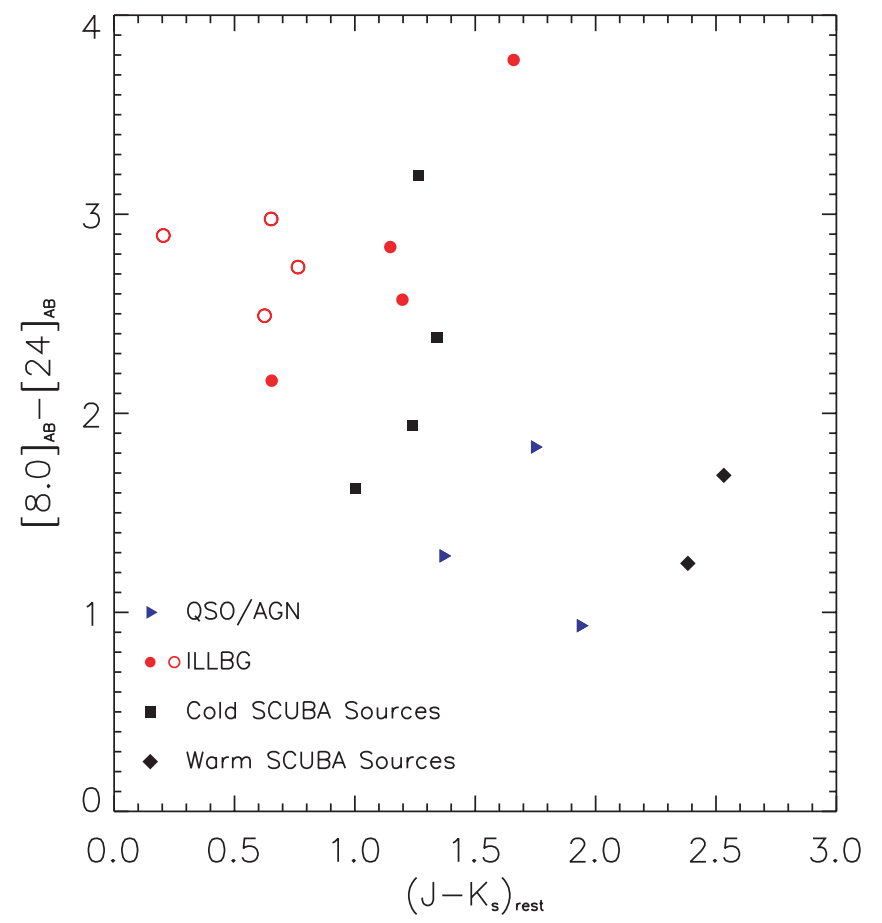

FIG. 3.-Observed-frame [8.0] - [24] vs. rest-frame $J-K_{s}$ color-color diagram for ILLBGs, AGNs, and SCUBA sources. At $z=3$, observed [4.5] - [8.0] is nearly equivalent to rest-frame $J-K_{s}$, and we have converted to $J-K_{s}$ using $K$-corrections from Hogg et al. (2002). The $J-K_{s}$ color is in Vega magnitudes for comparison with the 2MASS results (Cutri et al. 2001; Cole et al. 2001), but $[8.0]-[24]$ is in AB magnitudes.

attenuation can reproduce almost all the LBG colors. Shapley et al. (2001), Papovich et al. (2001), and Labbé et al. (2005) argue that a model with constant star formation rate fits the SEDs of near-infrared bright LBGs better. This model should also be very close to the real star formation history for the $8 \mu \mathrm{m}$ bright LBGs and ILLBGs. As shown in Figure $2, g^{\prime}-R$ is more sensitive to dust extinction, and $R-[3.6]$ is sensitive to both age and dust extinction for galaxies with a constant star formation history. A rough estimate of the extinction range as $0.1<$ $E(B-V)<0.4$ is consistent with what Shapley et al. (2001) and Papovich et al. (2001) measured in their LBG samples. The LBGs with $8 \mu \mathrm{m}$ detections tend to be older but little dustier than those not detected at $8 \mu \mathrm{m}$. The ILLBGs are more extreme; most are very red in $g^{\prime}-R$ and $R-[3.6]$, some beyond the model prediction. Shapley et al. (2001) also found that the SEDs of LBGs with the reddest $R-K_{S}$ defied any simple model fitting and argued that those are starburst galaxies with multicomponent morphologies, each with different colors. Such a red color could also be due to a significant contribution from an obscured AGN, but we argue below that significant AGN emission is inconsistent with the $[4.5]-[8.0]$ colors.

\section{ORIGIN OF $24 \mu \mathrm{m}$ EMISSION FROM ILLBGs}

The observed $24 \mu \mathrm{m}$ emission ( $\approx 6 \mu \mathrm{m}$ rest wavelength) from the ILLBGs can come from either the power-law component of an AGN (e.g., Elvis et al. 1994; Egami et al. 2004; Stern et al. 2005 ) or from warm dust heated by a starburst (e.g., Sanders \& Mirabel 1996; Rigopoulou et al. 1999). For strong starbursts, the $6.2 \mu \mathrm{m}$ PAH feature can enhance the observed flux by perhaps a factor of 1.5 (Tran et al. 2001; Peeters et al. 2004). As Ivison et al. (2004) point out, a key diagnostic for distinguish- ing between starbursts and AGNs is the existence of a change of slope around rest 3-4 $\mu \mathrm{m}$ : strong AGNs have nearly a constant slope from 2 to $10 \mu \mathrm{m}$, whereas starbursts show a distinct minimum in the $3-4 \mu \mathrm{m}$ range. Translated to an observational criterion, rest $J-K_{s}>2$ implies an AGN (Cutri et al. 2001; cf. Fig. 3 of Ivison et al. 2004).

Figure 3 shows the [8.0]-[24] versus $J-K_{s}$ color-color diagram for starburst-AGN separation suggested by Ivison et al. (2004). QSOs and warm SCUBA sources exhibit rest-frame $J-K_{s}$ color as red as local AGNs (Cutri et al. 2001), while all ILLBGs except one have $J-K_{s}<1.5$. Therefore, we suggest that the $24 \mu \mathrm{m}$ flux densities of ILLBGs are mainly due to strong dust emission caused by intensive star formation. ILLBGs have much redder [8.0]-[24] colors than QSOs and AGNs, steeper than typical power-law SEDs but consistent with strong starbursts. The SCUBA sources occupy the same overall portion of color-color space in this plot as do ILLBGs, AGNs, and QSOs at $z \sim 3$. In other words, the cold SCUBA sources appear to be Arp 220-like starburst galaxies at the upper left corners, and the warm SCUBA sources are AGNs. We conclude that ILLBGs, like cold SCUBA sources, are starburst galaxies.

\section{CHARACTERIZING THE GALAXY POPULATION AT $z=3$}

The faint blue LBGs that lack $24 \mu \mathrm{m}$ detections could have dust emission with flux densities below our detection threshold. For example, even blue dwarf galaxies are found to have dust emission at rest-frame $6 \mu \mathrm{m}$, presumably with PAH features (Rosenberg et al. 2006). We used a stacking technique to assess the average dust emission for the blue LBG population at $z=3$. The LBGs without $24 \mu \mathrm{m}$ detection were divided into two groups for stacking: those with $8 \mu \mathrm{m}$ detections and those without. There are a total of 198 LBGs lacking $24 \mu \mathrm{m}$ detections, including both spectroscopically identified and unidentified ones. In this subset, $31 \mathrm{LBGs}$ have $8 \mu \mathrm{m}$ detections. The 8 and $24 \mu \mathrm{m}$ images for both groups were stacked separately.

The stacking results show a significant difference between LBGs with and without $8 \mu \mathrm{m}$ detections. The LBGs without $8 \mu \mathrm{m}$ detections have $\left\langle M_{K}(\mathrm{Vega})\right\rangle=-21.5$, right in the range exhibited by local dwarf galaxies as shown in Figure 4. The upper limit in the [8.0]-[24] colors is also consistent with colors of local blue dwarf galaxies. This strongly suggests that most faint blue LBGs in the sample have similar stellar masses, dust obscuration, and star-formation histories to dwarf galaxies in the local universe. The LBGs with $8 \mu \mathrm{m}$ detections, including the ILLBGs, have $\left\langle M_{K}\right.$ (Vega) $\rangle=-24.5$, brighter than the local $M_{K}^{*}($ Vega $)=-23.4+5 \log (h)($ Cole et al. 2001; Huang et al. 2003). The $\left\langle M_{K}\right.$ (Vega) $\rangle$ and $\langle[8.0]-[24]\rangle$ for the $8 \mu$ m-detected LBGs are only slightly fainter and bluer than those of ILLBGs, suggesting that they may be similar kinds of galaxies but a little fainter and bluer, just below the $24 \mu \mathrm{m}$ limiting flux density.

All the results show that the LBG population is far from uniform. Figures 1 and 4 demonstrate that the LBGs with the largest stellar luminosities, as indicated by $8 \mu \mathrm{m}$ flux densities, are older and dustier than the LBGs with lower stellar luminosities. A larger LBG sample with infrared photometry and deeper $8 \mu \mathrm{m}$ observations is needed to tell whether there are two distinct populations or a continuous range of galaxy properties.

D. Rigopoulou et al. (2005, in preparation) conducted a detailed analysis of the stellar populations and estimated stellar masses for the $8 \mu \mathrm{m}$-selected LBGs. All were found to be massive galaxies $\left(>4 \times 10^{10} M_{\odot}\right)$, with the ILLBGs tending to have higher masses $\left(>10^{11} M_{\odot}\right)$. These masses are consistent with the mean 


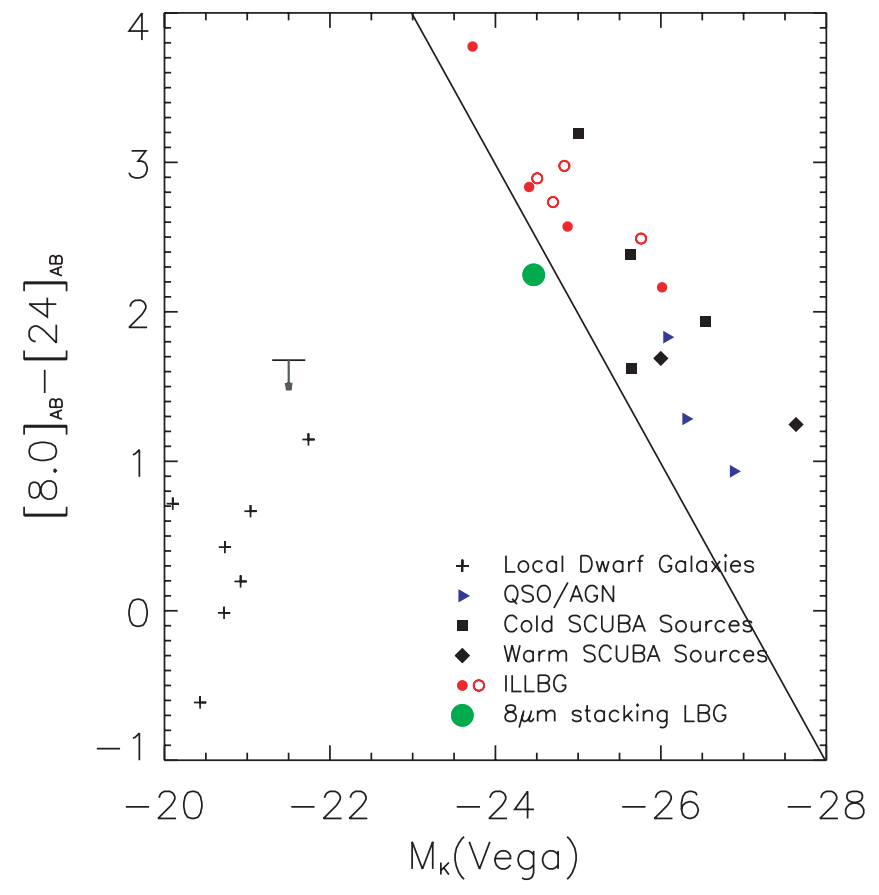

FIG. 4.-Observed-frame [8.0] - [24] vs rest-frame $M_{K}$ (Vega) color-magnitude diagram for ILLBGs, AGNs, and SCUBA sources. We derived the observed $8 \mu \mathrm{m}$ to rest- $K$-band $K$-correction with a spiral SED for ILLBGs and cold SCUBA sources and an AGN SED for AGNs, QSOs, and warm SCUBA sources. A mean redshift of $z=2.97$ for LBGs was assigned to those LBGs without spectroscopic redshifts. The upper limit shown at $M_{K}($ Vega $)=-21.5$ is for the stacked LBGs lacking $8 \mu \mathrm{m}$ detections. The green filled circle is for the stacked LBGs having $8 \mu$ m detections. For objects at $z \sim 3,[8.0]-[24]$ color is equivalent to the rest-frame color $K-[6.0]$. We plot the $K-[5.8]$ color for local blue dwarf galaxies (J. Rosenberg et al. 2005 , in preparation) for comparison. The solid line is the MIPS $24 \mu \mathrm{m}$ limiting flux density for galaxies at $z=3$.

dynamic mass of $1.5 \times 10^{11} M_{\odot}$ for starburst-type SCUBA galaxies (Swinbank et al. 2004; Tecza et al. 2004). Massive galaxies with $10^{11} M_{\odot}$ have also been found at $1 \lesssim z \lesssim 2$ (Cimatti et al. 2002; Daddi et al. 2004; Saracco et al. 2004; Glazebrook et al. 2004; Tecza et al. 2004; Labbé et al. 2005). These observations challenge current theoretical predictions of galaxy formation (Kauffmann et al. 1999; Shu et al. 2001; Baugh et al. 2003; Somerville et al. 2004). This study provides further confirmation that at least some massive galaxies were formed by $z=3$, favoring galaxy formation and evolution models assuming more rapid stellar mass accumulation scenarios (Nagamine et al. 2005).

\section{CONCLUSIONS}

This infrared study of LBGs reveals that they are a diverse group in terms of both their masses and dust contents. The observed $8 \mu \mathrm{m}$ (rest $2 \mu \mathrm{m}$ ) flux densities, which are roughly proportional to stellar mass, extend over at least 1.5 orders of magnitude, and the ratio of 24 to $8 \mu \mathrm{m}$ flux densities, which measure the fraction of ISM emission, extends over more than an order of magnitude.

Among 253 LBGs in the EGS area covered by Spitzer, 5\% were detected with $f_{24} \mu \mathrm{m}>60 \mu \mathrm{Jy}$. We refer to these as ILLBGs. ILLBGs have much redder observed $R-[3.6]$ colors than the other LBGs in the sample. The rest-frame $J-K_{s}$ colors for most ILLBGs are stellar, with no sign of an AGN power-law component. Therefore, we suggest that the $24 \mu \mathrm{m}$ emission is dominated by dust heated in a massive starburst, probably including some contribution from the PAH feature at rest-frame $6.2 \mu \mathrm{m}$. ILLBGs and cold SCUBA sources share the same infrared properties, suggesting that they are closely related and may belong to the same population. If so, it ought to be possible to detect submillimeter emission from ILLBGs. Both ILLBGs and cold SCUBA sources tend to be massive, with a typical stellar mass of $10^{11} M_{\odot}$. Such masses make them candidates for the progenitors of present-day giant ellipticals.

In contrast to ILLBGs, most LBGs in the sample are only detected at 3.6 and $4.5 \mu \mathrm{m}$ and have faint mid-infrared luminosities and blue $R-[3.6]$ colors. Their lower average $8 \mu \mathrm{m}$ flux density measured with the stacked image implies that the faint LBGs have much less stellar mass than ILLBGs, in a range similar to local dwarf galaxies. An upper limit of [8.0] - [24] color for average faint LBGs is also consistent with the dust emission of local dwarf galaxies.

J. L. R. has received support from an NSF Astronomy and Astrophysics Postdoctoral Fellowship under grant AST-0302049. This work is based on observations made with the Spitzer Space Telescope, which is operated by the Jet Propulsion Laboratory, California Institute of Technology, under NASA contract 1407. Support for this work was provided by NASA through contract 1256790, issued by JPL.
Barmby, P., et al. 2004, ApJS, 154, 97

Baugh, C. M., Benson, A. J., Cole, S., Frenk, C. S., \& Lacey, C. 2003, in Proc. ESO Workshop, The Mass of Galaxies at Low and High Redshift, ed. R. Bender \& A. Renzini (Berlin: Springer), 91

Bundy, K. et al. 2005, ApJ, 625, 621

Calzetti, D., et al. 2000, ApJ, 533, 682

Chapman, S. C., Blain, A. W., Ivison, R. J., \& Smail, I. R. 2003, Nature, 422, 695

Chapman, S. C., et al. 2005, ApJ, 622, 772

Cimatti, A., et al. 2002, A\&A, 391, L1

Cole, S., et al. 2001, MNRAS, 326, 255

Cutri, R., et al. 2001, in ASP Conf. Ser. 232, New Era of Wide Field Astronomy, ed. R. Clowes, A. Adamson, \& G. Bromage (San Francisco: ASP), 78

Daddi, E., et al. 2004, ApJ, 600, L127

Drory, N., et al. 2004, ApJ, 608, 742

Egami, E. et al. 2004, ApJS, 154, 130

Elvis, M. et al. 1994, ApJS, 95, 1

Fazio, G. G. et al. 2004a, ApJS, 154, 10 2004b, ApJS, 154, 39

Glazebrook, K., et al. 2004, Nature, 430, 181

Hogg, D., et al. 2002, preprint (astro-ph/0210394)

\section{REFERENCES}

Huang, J.-S., Barmby, P., et al. 2004, ApJS, 154, 44

Huang, J.-S., Glazebrook, K., Cowie, L. L., \& Tinney, C. 2003, ApJ, 584, 203

Ivison, R. J., Greve, T. R., Smail, I., et al. 2002, MNRAS, 337, 1

Ivison, R. J., et al. 2004, ApJS, 154, 124

Kauffmann, G., et al. 1999, MNRAS, 303, 188

Labbé, I., et al. 2005, ApJ, 624, L81

Nagamine, K., et al. 2005, ApJ, 627, 608

Papovich, C., Dickinson, M., \& Ferguson, H. C. 2001, ApJ, 559, 620

Peeters, E., et al. 2004, ApJ, 613, 986

Rigopoulou, D., et al. 1999, AJ, 118, 2625

Rosenberg, J., et al. 2006, ApJ, in press

Sanders, D., \& Mirabel, I. F. 1996, ARA\&A, 34, 749

Saracco, P., et al. 2004, A\&A, 420, 125

Shapley, A. E., Steidel, C. C., Adelberger, K. L., Dickinson, M., Giavalisco, M., \& Pettini, M. 2001, ApJ, 562, 95

Shu, C. G., Mao, S., \& Mo, H. J. 2001, MNRAS, 327, 895

Simpson, C., Dunlop, J. S., Eales, S. A., Ivison, R. J., Scott, S. E., Lilly, S. J., \& Webb, T. M. A. 2004, MNRAS, 353, 179

Smail, I., Ivison, R. J., Blain, A. W., \& Kneib, J.-P. 2002, MNRAS, 331, 495

Somerviller, R., et al. 2004, ApJ, 600, L135 
Steidel, C. C., Adelberger, K. L., Shapley, A. E., Pettini, M., Dickinson, M., \& Giavalisco, M. 2003, ApJ, 592, 728

Steidel, C. C., et al. 2004, ApJ, 604, 534

Stern, D., et al. 2005, ApJ, 631, 163

Swinbank, A. M., Smail, I., Chapman, S. C., Blain, A. W., Ivison, R. J., \& Keel, W. C. 2004, ApJ, 617, 64
Tecza, M., et al. 2004, ApJ, 605, L109

Tran, Q. D., et al. 2001, ApJ, 552, 527

Webb, T. M. A., et al. 2003, ApJ, 597, 680

Wilson, G., Huang, J.-S., et al. 2004, ApJS, 154, 107 